TFIIS and strandtransfer proteins

SIR - Davies et al. ${ }^{1}$ recently pointed out the sequence similarity between the yeast PPR2 protein ${ }^{2}$, which is required for transcriptional induction of the $U R A 4$ gene, and transcription elongation factor SII (also called TFIIS) ${ }^{3}$. Since then, the sequence of DST1 (ref. 4) has been published and is identical to PPR2. DST1 encodes DNA-strand transfer protein- $\alpha$, which has $\operatorname{RecA}$-like activity in vitro in that it can promote the transfer of one strand of a doublestranded DNA molecule to a homologous single strand, and thus may be involved in recombination ${ }^{5,6}$.

It had seemed likely that $P P R 2$ encoded a TFIIS-related protein rather than TFIIS itself, PPR2 being much smaller than mouse TFIIS (ref. 7). However, this conclusion was based on the original PPR2 sequence ${ }^{2}$, which has minor differences from DST1. The DST1 sequence now predicts a 309 amino-acid protein of relative molecular mass 34,800 (ref. 4), similar in size to mouse TFIIS (301 residues) and the biochemically identified yeast TFIIS protein $^{8}$. Sequence similarity between the DST1(PPR2) protein and mouse TFIIS extends throughout most of its length (see figure). As with TFIIS from other species, there is a central, poorly conserved domain.

TFIIS allows RNA polymerase II in vitro to pass certain DNA sequences which would otherwise cause the transcription complex to pause on the template, and it is conceivable that strand transfer activity is relevant to this activity. We speculate that TFIIS discourages pausing by a direct effect on the template, perhaps by helping to denature the untranscribed DNA. Alternatively, if pausing is governed by the hybrid RNADNA duplex made at the pause site, the strand transfer activity might encourage elongation by promoting the displace-

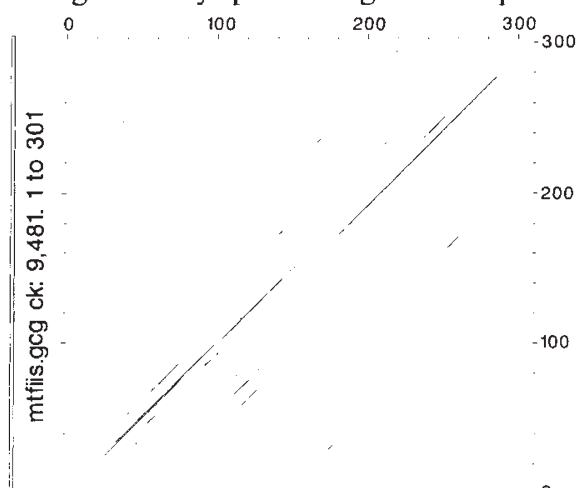

dstipro.gcg ck: $3,634,1$ to 309

Dot-plot comparison of yeast PPR2/DST1 (horizontal axis) and mouse TFIIS (vertical axis) amino acid sequences (window 50, stringency 19). ment of the transcript and renaturation of the template (although the transfer activity has not been shown to be active on RNA-DNA duplexes).

PPR2/DST1 now seems an excellent candidate for yeast TFIIS, although the intriguing possibility remains that they are two distinct but related proteins. It is important to demonstrate whether purified TFIIS has strand-transfer activity, as predicted. Curiously, DSTI overproduced in $E$. coli does not house strandtransfer activity ${ }^{4}$, possibly because of the need for accessory factors or secondary modification. The mutants $p p r 2 / d s t 1$ are unable to induce transcription of the URA4 gene ${ }^{2}$ and show a meiotic (but not mitotic) recombination defect $^{4}$. Clearly following the in vitro assays with in vivo studies such as these are needed to define the role of this protein in the cell.

MRC Human Genetics Unit,

DAVID KIPLING

Western General Hospital,

Crewe Road,

Edinburgh EH4 $2 X U$, UK

Department of Zoology,

Stephen E. KeARSEY

University of Oxford,

South Parks Road, Oxford OX1 3PS, UK

1. Davies, C. J., Trgovcich, J. \& Hutchinson, C. A. Nature 345, 298-298 (1990)

2. Hubert, J-C. et al. EMBO J. 2, 2071-2073 (1983)

3. Hirashima, S., Hirai, H., Nakanishi, Y. \& Natori, S. J. Biol. Chem. 263, 3858-3863 (1988)

4. Clark, A. B., Dykstra, C. C. \& Sugino, A. Molec. cell. Biol. 11, 2576-2582 (1991)

5. Sugino, A., Nitiss, J. \& Resnick, M. A. Proc. nat. Acad Sci. U.S.A. 85, 3683-3687 (1988)

6. Hamatake, R. K., Dykstra, C. C. \& Sugino, A. J. biol. Chem. 264, 13336-13342 (1989).

7. Marshal!, T. K., Guo, H. \& Price, D. H. Nucleic Acids Res. 18, 6293-6298 (1990).

8. Sawadogo, M., Sentenac, A. \& Fromageot, P. J. biol Chem. 255, 12-15 (1980)

\section{Premature death}

SIR - Williams et al. ${ }^{1}$ report mass mortalities of black sea urchins (Diadema antillarum) in the Florida Keys and the Caribbean Sea. Their report is based on uncoordinated, qualitative or unverified observations, unlike the painstakingly assembled observations of the first reported mass mortality of this species in 1983-84 (ref. 2).

Although black sea urchins have been observed dying at several sites in the Keys and, apparently, elsewhere, Williams et al. make some misleading points: (1) that sea urchins are dying in the same numbers as in $1983-84$; (2) that the time course for the mortality at each site is slower than for the original 198384 mass mortality; (3) that a potential class of pathogens has been identified; (4) that there is an implied correlation with coral bleaching; and (5) that a link is implied to an alleged mass mortality of another species of urchin in Puerto Rico. These points are not documented, and the request for additional observations and reports can only further confuse the situation.

Williams has established an information-clearing centre to collect observations and reports of marine phenomena and disturbances over broad geographical regions. Such information must be carefully interpreted before it is reported to the community at large. Full understanding of the causes, magnitude and impact of regional events such as a mass mortality of sea urchins will come only from coordinated observation networks using standardized observation protocols.

JOHN C. OGDEN

Florida Institute of Oceanography,

University of South Florida,

830 First Street South,

St Petersburg,

Florida 33701, USA

1. Williams, E.H. Jr et al. Nature 352, 385 (1991).

2. Lessios, H.A., Robertson, D.R. \& Cubit, J.D. Science 226. 335-337 (1984).

\section{Break for SCIDs}

SIR - Following our report of the successful engraftment of human peripheral blood leukocytes into severe combined immunodeficient (SCID) mice ${ }^{1}$, we published a brief note ${ }^{2}$ acknowledging that we had overestimated the number of human cells. We and others ${ }^{3-5}$ have now studied many hu-PBL-SCID mice, and we find that human cells comprise most cells recovered from the peritoneal cavity, but a small minority (1-5\%) of cells in lymphoid tissues or peripheral blood. The presence of human cells in lymph nodes and blood for at least 24 weeks after PBL injection was confirmed by PCR amplification of human genes. These data reconfirm the long-term persistence of human cells in hu-PBL-SCID mice, but in smaller numbers than we originally reported ${ }^{1}$.

DONALD E. MOSIER

RICHARD J. GULIZIA

BRUCE E. TORBETI

Medical Biology Institute,

11077 North Torrey Pines Road,

La Jolla, California 92037, USA

STEPHEN M. BAIRD

Veteran's Administration Medical

Center,

Department of Pathology,

University of California,

San Diego, La Jolla,

California 92161, USA

La Jolla Institute of Experimental Medicine,

La Jolla, California 92037, USA

\footnotetext{
1. Mosier, D. E., Gulizia, R. J., Baird, S. M. \& Wilson, D. B. Nature 335, 256-259 (1988)

2. Mosier, D. E., Gulizia, R. J., Baird, S. M. \& Wilson, D. B. Nature 338, 211 (1989).

3. Krams, S. M., Dorshkind, K. \& Gershwin, M. E. J. exp. Med. 170, 1919 (1989).

4. Duchosal, M. A. et al. J. exp. med. 172, 985 (1989). 5. Saxon, A. et al. J. clin. Invest. 87, 658 (1991).
} 